Review began 02/13/2022 Review ended 03/02/2022 Published 03/04/2022

๑) Copyright 2022

Yanamandra et al. This is an open access article distributed under the terms of the Creative Commons Attribution License CCBY 4.0., which permits unrestricted use, distribution, and reproduction in any medium, provided the original author and source are credited.

\section{Relationship of Computed Tomography Severity Score With Patient Characteristics and Survival in Hypoxemic COVID-19 Patients}

Uday Yanamandra $^{1}$, Shivendra Shobhit ${ }^{2}$, Debashish Paul ${ }^{2}$, Bhavya Aggarwal ${ }^{3}$, Praneet Kaur ${ }^{3}$, Gayatri Duhan $^{3}$, Anurag Singh ${ }^{3}$, Rajagopal Srinath ${ }^{1}$, Puneet Saxena ${ }^{4}$, Anil S. Menon ${ }^{1}$

1. Internal Medicine, Armed Forces Medical College, Pune, IND 2. Anesthesiology and Critical Care, Armed Forces Medical College, Pune, IND 3. General Practice, Armed Forces Medical Services, New Delhi, IND 4. Pulmonology, Army Hospital (Research And Referral; R\&R), New Delhi, IND

Corresponding author: Puneet Saxena, drsaxenap@hotmail.com

\section{Abstract \\ Background}

Computed tomography (CT) scans and CT severity scores (CTSS) are widely used to assess the severity and prognosis in coronavirus disease 2019 (COVID-19). CTSS has performed well as a predictor in differentiating severe from non-severe cases. However, it is not known if CTSS performs similarly in hospitalized severe cases with hypoxia at admission.

\section{Methods}

We conducted a retrospective comparative study at a COVID-care center from Western India between 25th April and 31st May 2021, enrolling all consecutive severe COVID-19 patients with hypoxemia (peripheral oxygen saturation < 94\%). Neutrophil-lymphocyte ratio (NLR), C-reactive protein (CRP), interleukin-6 (IL-6), lactate dehydrogenase (LDH), D-dimer, ferritin, and CT thorax were done within $24 \mathrm{~h}$ of admission before being initiated on any anti-COVID-19 therapy. CTSS was calculated by visual assessment and categorized into three severity categories and was correlated with laboratory markers and overall survival (OS). Statistical analysis was done using John's Macintosh Project (JMP) 15.0.0 ver. 3.0.0 (Cary, North Carolina).

\section{Results}

The median age of the study population (n-298) was 59 years (24-95) with a male preponderance $(61.41 \%$, $\mathrm{n}=183$ ). The 15 and 30-day survivals were $67.64 \%$ and $59.90 \%$, respectively. CTSS did not correlate with age, gender, time from vaccination, symptoms, or comorbidities but had a significant though weak correlation with LDH ( $p=0.009)$, D-dimer ( $p=0.006)$, and NLR $(p=0.019)$. Comparing demographic and laboratory aspects using CT severity categories, only NLR $(\mathrm{p}=0.0146)$ and $\mathrm{D}$-dimer $(\mathrm{p}=0.0006)$ had significant differences. The $15 \mathrm{~d}$-OS of mild, moderate, and severe CT categories were $88.62 \%$, $70.39 \%$, and $52.62 \%$, respectively, while 30d-OS of three categories were $59.08 \%, 63.96 \%$, and $49.12 \%$, respectively.

\section{Conclusion}

Among hospitalized severe COVID-19 patients with hypoxemia at admission, CT severity categories correlate well with outcomes but not inflammatory markers at admission.

Categories: Radiology, Infectious Disease, Pulmonology

Keywords: crp, neutrophil-lymphocyte ratio, d-dimer, ldh, inflammatory markers, ct severity score, computed tomography, covid-19

\section{Introduction}

Coronavirus disease 2019 (COVID-19), caused by the novel severe acute respiratory syndrome coronavirus 2 (SARS-CoV-2), has had a devastating impact on the global population in the last two years. A computed tomography scan of the thorax (CT thorax) in high-resolution CT protocol (HRCT) gives valuable information about the extent and stage of the disease and evaluates pre-existing/co-existing cardio-thoracic abnormalities that may affect the management plan and prognostication [1-3]. Since the start of the pandemic, there has been an ongoing debate on the indications, timing, and utility of CT thorax in COVID19 with recommendations now favoring this imaging modality in moderate to severe disease [4]. It has previously been shown that the extent of changes seen on CT thorax correlates well with the severity of disease, outcomes, and inflammatory markers [5-10]. Patients with severe disease are likely to have higher CT severity scores (CTSS) than those with mild illnesses [11]. CTSS is better associated with hospital admission and intensive care unit (ICU) requirements than with mortality [9]. Among hospitalized patients, survivors have been found to have a lower CTSS compared to non-survivors [12]. However, $40 \%$ of subjects in the aforementioned study did not have hypoxia at admission. It is not clear whether CT thorax can be a 
prognostic marker among patients who present with severe disease with hypoxia. We conducted a retrospective study in a cohort of hospitalized severe COVID-19 cases to see the correlation of CT thorax with outcomes and laboratory parameters.

\section{Materials And Methods}

\section{Study design}

The study was a retrospective, observational cross-sectional study conducted at a COVID-care center from 25th April to 31st May 2021. This center catered for reverse transcriptase-polymerase chain reaction (RTPCR)-positive severe-COVID cases. Severity was defined by pulse oximeter oxygen saturation (SpO2) level at room air of $<90 \%$, or respiratory rate $>30$ breaths per minute at initial presentation. Hypoxemia was defined as $\mathrm{SpO} 2<94 \%$. The institutional ethical committee approved the study, and informed consent was obtained for all the patients. The study was done in accordance with the Declaration of Helsinki.

\section{Subjects}

All consecutive patients admitted to this study center were screened for inclusion in the study. During the study period, there was a massive surge of COVID-19 cases and there was a shortage of ICU beds and trained staff. It was the institutional protocol to subject all severe COVID-19 cases to HRCT chest. This was done to assist in triaging, allocate ICU beds, and expedite evaluation for other co-existing lung conditions that needed attention. Thus, all severe cases were subjected to HRCT chest unless they were too unstable for undergoing a CT scan. All those patients who had severe disease and underwent HRCT scanning within $24 \mathrm{~h}$ of admission and at least four days post-development of any of the COVID symptoms were included in the study. Patients who had already been started on glucocorticoids were not included for analysis. All initial chest HRCT scans were performed on the day of the patients' presentation using a volume computed tomography (VCT) GE 64 scanner (General Electric Company, Boston, Massachusetts). The supine position was adopted for scans with a single breath-hold. Contiguous image acquisition was carried out, and the images were subsequently reconstructed into a lung window in the sharp algorithm (60-80 kernel) and a soft tissue window (20-30 kernel) with a section thickness of $1.5 \mathrm{~mm}$. Radiologists with more than eight years of experience studied the scans for qualitative and quantitative assessment in each patient. The scans were assessed for the presence or absence of typical findings of COVID-19 pneumonia as defined by the Radiological Society of North America (RSNA) consensus statement $[1,13]$. CTSS was then calculated using the visual assessment of each lobe involved [14-15]. Each lobe was evaluated for the proportion of lobe evaluated and scored as $0,1,2,3,4$, or 5 depending on the involved proportion of $0 \%,<5 \%, 5-25 \%, 26-50 \%$, 51 to $75 \%$, and greater than $75 \%$, respectively. The scores of each of the five lobes were totaled to calculate the total CT severity score $[5-6,16]$. These patients were further classified based on their CT severity score as mild ( $\leqslant 7)$, moderate (7-17), and severe cases (>17) [6]. Those patients who were unable to undergo CT scan for any medical contraindication, hemodynamic instability, or were unwilling to undergo HRCT Chest were excluded.

\section{Methodology}

A detailed history regarding comorbidities (hypertension, cardiovascular diseases, respiratory disorders, hypothyroidism, and central nervous system (CNS) disorders), COVID vaccination status, days from last vaccination, and COVID symptomatology (fever, dyspnea, cough, expectorations, hemoptysis, myalgia, headache, chest pain, ageusia, and diarrhea) were recorded at the time of admission along with the medical history of the patient. Neutrophil-lymphocyte ratio (NLR), C-reactive protein (CRP), interleukin-6 (IL-6), lactate dehydrogenase (LDH), D-dimer, and ferritin were measured at admission and within 24 hours of HRCT chest. All the patients were managed with currently prevailing protocol-based therapy guided by national guidelines, including steroids, low molecular weight heparin (LMWH), and vitamin and mineral supplements. Discharge criteria were uniformly applied as per the prevalent local guidelines. The outcome parameters included days of hospitalization and mortality.

\section{Statistical analysis}

The data were analyzed using John's Macintosh Project (JMP) 15.0.0 ver. 3.0.0 (Cary, North Carolina). The continuous data were assessed for normal distribution. Bivariate linear fit and polynomial fit was used to analyze the correlation between the continuous variables, whereas analysis of variance (ANOVA) was used to analyze the correlation of a continuous variable with nominal variables. The whole model test for logistic fit was done using the chi-square test $(\chi 2)$. The continuous variables were represented as median (Range; Mean \pm SD). $p<0.05$ was considered significant. The survival analysis was done using the Kaplan-Meier method. The log-rank test was used to estimate significance. During analysis, the CT scores were converted to a scale of 0-1 for better interstudy comparison, as internationally, authors used different CTSS scores ranging from $0-15,0-20$ to $0-75$.

\section{Data}

The data are stored as de-identified participant data which are available on reasonable request to the authors. 


\section{Cureus}

\section{Results}

Among the screened population of 357 patients, HRCT was done in 298 patients (study population, 83\%) within 24 hours of admission and more than four days of the symptom's onset (Figure 1). The median age of the study population was 59 years $(24-95 ; 58.01 \pm 14.89)$ with a male preponderance $(61.41 \%, \mathrm{n}=183)$. The most common symptoms in the study population were dyspnea, cough, and fever in $73.83 \%$ (n-220), $72.15 \%$ (n-215), and 58.05\% (n-173), respectively. Vaccination by COVISHIELD ${ }^{\circledR}$ (Serum Institute of India Pvt Ltd, New Delhi, India) was carried out in 65 (21.81\%) patients, of which 13 (4.36\%) had received both doses of vaccine. The median time from the last dose of vaccination to hospital admission was 24 days $(0-66$; $25.46 \pm 13.45)$. The distribution of the comorbidities and symptoms among the study population is tabulated in Table 1. The distribution of the CT scores among the study population is depicted in Figure 1 and Figure 2. The median duration of hospital stay among the study population was nine days $(0-37 ; 12.2 \pm 8.9)$. A total of 80 patients succumbed to the illness, with $26.85 \%$ mortality. The 15 and 30 -day survival of the study population was $67.64 \%$ and $59.90 \%$.

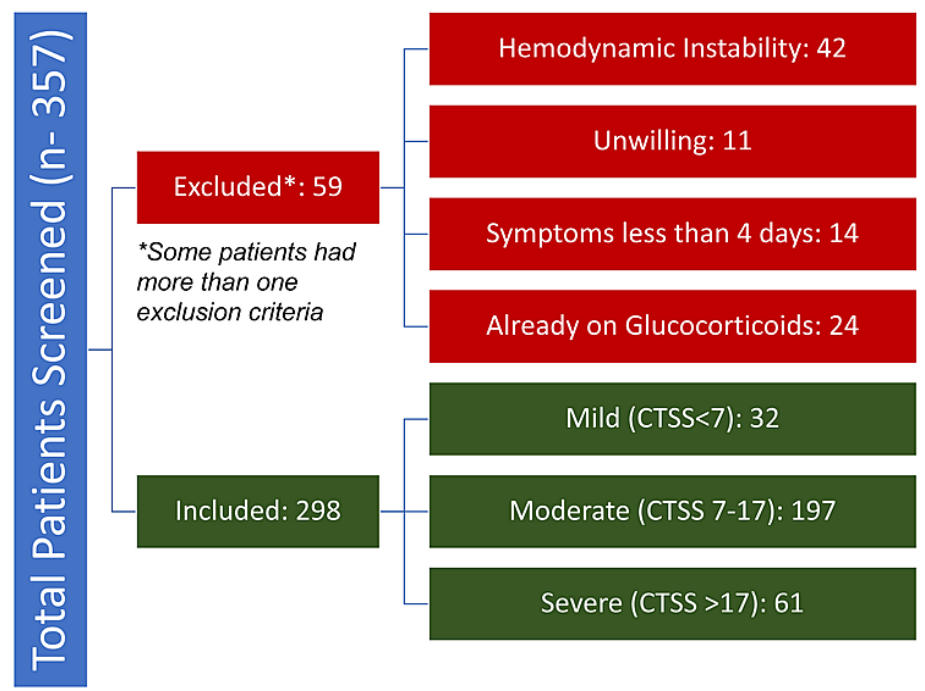

FIGURE 1: Consort diagram

CTSS: computed tomography severity score 


\section{Cureus}

\begin{tabular}{|c|c|c|}
\hline Presence of symptoms/co-morbidities & $\mathbf{n}$ & $\%$ \\
\hline Diabetes mellitus & 112 & 37.58 \\
\hline Hypertension & 119 & 39.93 \\
\hline Cerebrovascular disease & 11 & 3.69 \\
\hline Fever & 173 & $58.05 \%$ \\
\hline Cough & 215 & $72.15 \%$ \\
\hline Expectoration & 3 & $1.01 \%$ \\
\hline Dyspnea & 220 & $73.83 \%$ \\
\hline Myalgia & 41 & $13.76 \%$ \\
\hline Diarrhea & 11 & $3.69 \%$ \\
\hline Chest pain & 15 & $5.03 \%$ \\
\hline Headache & 23 & $7.72 \%$ \\
\hline Anosmia & 1 & $0.34 \%$ \\
\hline Sore throat & 5 & $1.68 \%$ \\
\hline Hemoptysis & 3 & $1.01 \%$ \\
\hline
\end{tabular}

TABLE 1: Symptoms and comorbidities of the study population 


\section{Cureus}

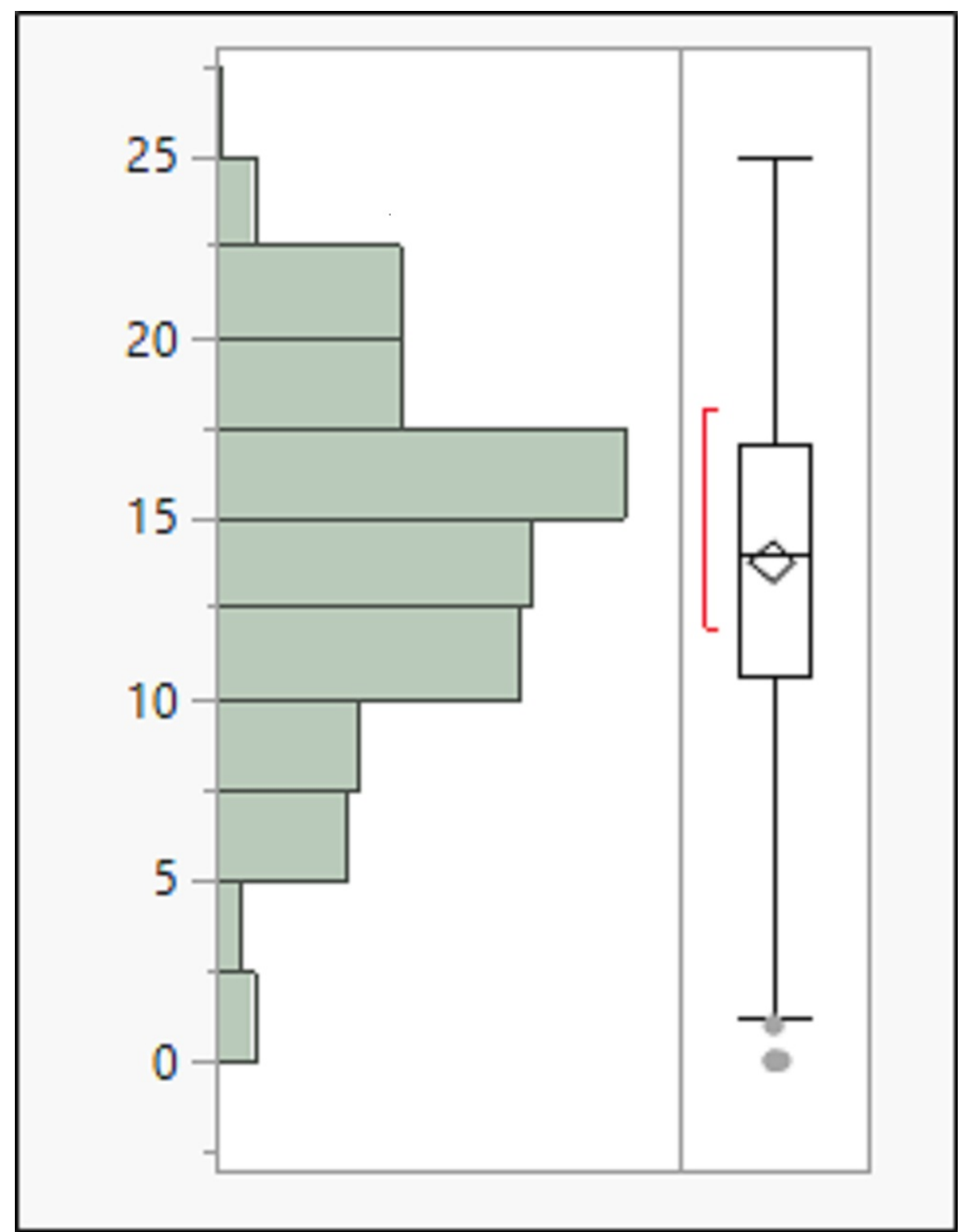

FIGURE 2: Distribution of CTSS among the study population

CTSS: computed tomography severity score

The CT score of the patients did not correlate with age, gender, and time from vaccination. There was no statistically significant correlation of any of the symptoms of COVID with CT scores. Vaccinated patients had lower CT scores than those who received no vaccination, but the difference was not statistically significant. None of the comorbidities had any correlation with the CT scores (Table 2). On analyzing the relation of various inflammatory markers and CT scores, only LDH, D-dimer, and NLR had a significant correlation (p-0.009, p-0.006, p-0.014, respectively) (Figure 3, Table 3). 


\section{Cureus}

\begin{tabular}{|c|c|c|c|c|c|}
\hline Symptoms & & $\mathrm{n}$ & Median CT score & Mean CT score & $p$-value \\
\hline \multirow{2}{*}{ Fever } & Present & 173 & $0.56(0-0.92)$ & $0.55 \pm 0.19$ & \multirow{2}{*}{0.9647} \\
\hline & Absent & 125 & $0.56(0.44-1)$ & $0.55 \pm 0.19$ & \\
\hline \multirow{2}{*}{ Cough } & Present & 215 & $0.55(0-1)$ & $0.54 \pm 0.19$ & \multirow{2}{*}{0.2749} \\
\hline & Absent & 83 & $0.6(0.05-0.975)$ & $0.57 \pm 0.19$ & \\
\hline \multirow{2}{*}{ Expectoration } & Present & 3 & $0.675(0.5-0.72)$ & $0.63 \pm 0.12$ & \multirow{2}{*}{0.4403} \\
\hline & Absent & 295 & $0.56(0-1)$ & $0.55 \pm 0.19$ & \\
\hline \multirow{2}{*}{ Dyspnea } & Present & 220 & $0.56(0-1)$ & $0.55 \pm 0.19$ & \multirow{2}{*}{0.655} \\
\hline & Absent & 78 & $0.56(0-0.96)$ & $0.56 \pm 0.19$ & \\
\hline \multirow{2}{*}{ Myalgia } & Present & 41 & $0.6(0-0.875)$ & $0.54 \pm 0.2$ & \multirow{2}{*}{0.8284} \\
\hline & Absent & 257 & $0.56(0-1)$ & $0.55 \pm 0.19$ & \\
\hline \multirow{2}{*}{ Diarrhea } & Present & 11 & $0.64(0.04-0.875)$ & $0.57 \pm 0.23$ & \multirow{2}{*}{0.4866} \\
\hline & Absent & 287 & $0.56(0-1)$ & $0.55 \pm 0.19$ & \\
\hline \multirow{2}{*}{ Chest pain } & Present & 15 & $0.56(0.32-0.875)$ & $0.58 \pm 0.14$ & \multirow{2}{*}{0.5966} \\
\hline & Absent & 283 & $0.56(0-1)$ & $0.55 \pm 0.19$ & \\
\hline \multirow{2}{*}{ Headache } & Present & 23 & $0.55(0-0.875)$ & $0.51 \pm 0.24$ & \multirow{2}{*}{0.6473} \\
\hline & Absent & 275 & $0.56(0-1)$ & $0.55 \pm 0.19$ & \\
\hline \multirow{2}{*}{ Anosmia } & Present & 1 & 0.68 & 0.68 & \multirow{2}{*}{0.4088} \\
\hline & Absent & 297 & $0.56(0-1)$ & $0.55 \pm 0.19$ & \\
\hline \multirow{2}{*}{ Sore throat } & Present & 5 & $0.675(0.32-0.96)$ & $0.637 \pm 0.23$ & \multirow{2}{*}{0.4094} \\
\hline & Absent & 293 & $0.56(0-1)$ & $0.55 \pm 0.19$ & \\
\hline \multirow{2}{*}{ Hemoptysis } & Present & 3 & $0.56(0.55-0.68)$ & $0.6 \pm 0.07$ & \multirow{2}{*}{0.6614} \\
\hline & Absent & 295 & $0.56(0-1)$ & $0.55 \pm 0.19$ & \\
\hline \multirow{2}{*}{ Diabetes } & Present & 112 & $0.6(0.2-0.96)$ & $0.56 \pm 0.17$ & \multirow{2}{*}{0.4066} \\
\hline & Absent & 186 & $0.55(0-1)$ & $0.54 \pm 0.20$ & \\
\hline \multirow{2}{*}{ Hypertension } & Present & 119 & $0.55(0.05-0.95)$ & $0.55 \pm 0.19$ & \multirow{2}{*}{0.5714} \\
\hline & Absent & 179 & $0.56(0-1)$ & $0.55 \pm 0.19$ & \\
\hline \multirow{2}{*}{ Cerebrovascular disease } & Present & 11 & $0.625(0.28-0.8)$ & $0.60 \pm 0.15$ & \multirow{2}{*}{0.3221} \\
\hline & Absent & 287 & $0.56(0-1)$ & $0.55 \pm 0.19$ & \\
\hline
\end{tabular}

\section{TABLE 2: Correlation of the CT score and the symptoms/co-morbidities}

Severity was defined by pulse oximeter oxygen saturation $(\mathrm{SpO} 2)$ level at room air of $<90 \%$ or respiratory rate $>30$ breaths per minute at initial presentation. Hypoxemia was defined as $\mathrm{SpO} 2<94 \%$. 


\section{Cureus}

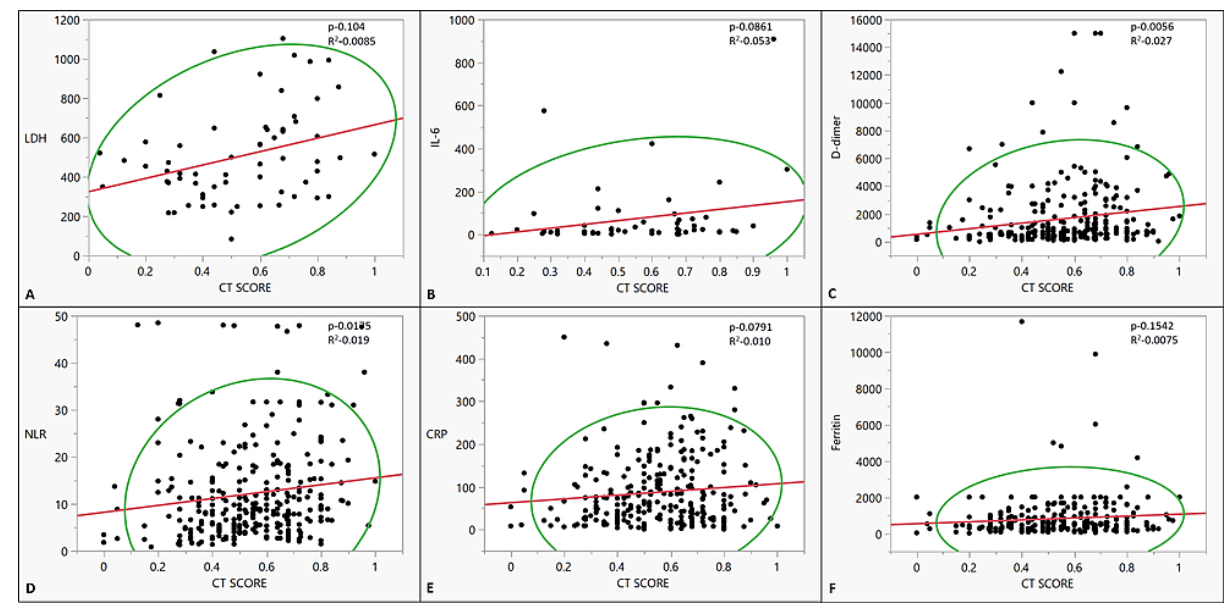

FIGURE 3: The co-relationship between $C T$ scores and inflammatory markers: (A) LDH (IU/L), (B) IL-6 (ng/dl), (C) D-dimer (ng/ml), (D) NLR, (E) CRP (mg/L), and (F) Serum ferritin (ng/ml)

CRP: C-reactive protein; NLR: neutrophilic lymphocytic ratio; IL-6: interleukin-6; LDH: lactate dehydrogenase; CT score: computed tomography score

Bivariate linear fit and polynomial fit were used to analyze the correlation and estimate significance. 


\section{Cureus}

\begin{tabular}{|c|c|c|c|c|}
\hline \multicolumn{3}{|l|}{ Characteristics } & $p$-value & $\mathbf{R}^{2}$ \\
\hline \multirow{3}{*}{ CRP } & $\mathrm{n}$ & 283 & \multirow{3}{*}{0.0791} & \multirow{3}{*}{0.010} \\
\hline & Median (IQR) & $63.51(0.56-450)$ & & \\
\hline & Mean \pm SD & $86.81 \pm 81.7$ & & \\
\hline \multirow{3}{*}{ LDH } & $\mathrm{n}$ & 65 & \multirow{3}{*}{0.0085} & \multirow{3}{*}{0.104} \\
\hline & Median (IQR) & 465(84-1103) & & \\
\hline & Mean \pm SD & $506.05 \pm 231.96$ & & \\
\hline \multirow{3}{*}{ IL-6 } & $\mathrm{n}$ & 56 & \multirow{3}{*}{0.0861} & \multirow{3}{*}{0.053} \\
\hline & Median (IQR) & 18.97(1.32-909) & & \\
\hline & Mean \pm SD & $74.41 \pm 155.35$ & & \\
\hline \multirow{3}{*}{ Serum Ferritin } & $\mathrm{n}$ & 271 & \multirow{3}{*}{0.1542} & \multirow{3}{*}{0.0075} \\
\hline & Median (IQR) & $480(11.7-11676)$ & & \\
\hline & Mean $\pm S D$ & $818.95 \pm 1162.99$ & & \\
\hline \multirow{3}{*}{ D-dimer } & $\mathrm{n}$ & 280 & \multirow{3}{*}{0.0056} & \multirow{3}{*}{0.027} \\
\hline & Median (IQR) & 708(3.5-15000) & & \\
\hline & Mean \pm SD & $1623.58 \pm 2334.67$ & & \\
\hline \multirow{3}{*}{ NLR } & $\mathrm{n}$ & 284 & \multirow{3}{*}{0.0175} & \multirow{3}{*}{0.019} \\
\hline & Median (IQR) & 8.92(0.83-48.48) & & \\
\hline & Mean $\pm S D$ & $12.20 \pm 9.98$ & & \\
\hline
\end{tabular}

\section{TABLE 3: Correlation of the CT score and inflammatory markers}

CRP: C-reactive protein; LDH: lactate dehydrogenase, IL-6: interleukin-6, NLR: neutrophil-lymphocyte ratio

The mean age was comparable in the three subcategories of CT severity (p-0.29). There was no statistically significant correlation with gender, vaccination, symptoms, and comorbidities between the three CT severity groups (Table 4). Only NLR and D-dimer were significantly different across categories, with severe categories having higher values $(\mathrm{p}<0.001 ; 0.015)$. There was no difference among the groups in other inflammatory markers (Figure 4, Table 5).

\begin{tabular}{|c|c|c|c|c|c|c|}
\hline Characteristics & & & Mild & Moderate & Severe & p-value \\
\hline \multirow{4}{*}{ Gender } & \multirow{2}{*}{ Male } & $\mathrm{n}$ & 19 & 120 & 44 & \multirow{4}{*}{0.8873} \\
\hline & & $\%$ & 6.38 & 40.27 & 14.77 & \\
\hline & \multirow{2}{*}{ Female } & $\mathrm{n}$ & 13 & 77 & 25 & \\
\hline & & $\%$ & 4.36 & 25.84 & 8.39 & \\
\hline \multirow{6}{*}{ Vaccination status } & \multirow{2}{*}{ Not vaccinated } & $\mathrm{n}$ & 21 & 157 & 55 & \multirow{6}{*}{0.3846} \\
\hline & & $\%$ & 7.05 & 52.68 & 18.46 & \\
\hline & & $\mathrm{n}$ & 10 & 31 & 11 & \\
\hline & & $\%$ & 3.36 & 10.40 & 3.69 & \\
\hline & \multirow{2}{*}{ Both Doses } & $\mathrm{n}$ & 1 & 9 & 3 & \\
\hline & & $\%$ & 0.34 & 3.02 & 1.01 & \\
\hline & & $\mathrm{n}$ & 10 & 76 & 26 & \\
\hline
\end{tabular}




\section{Cureus}

\begin{tabular}{|c|c|c|c|c|c|c|}
\hline \multirow{3}{*}{ Diabetes mellitus } & Present & $\%$ & 3.36 & 25.5 & 8.72 & \multirow{3}{*}{0.7244} \\
\hline & \multirow{2}{*}{ Absent } & $\mathrm{n}$ & 22 & 121 & 43 & \\
\hline & & $\%$ & 7.38 & 40.60 & 62.32 & \\
\hline \multirow{4}{*}{ Hypertension } & \multirow{2}{*}{ Present } & $\mathrm{n}$ & 13 & 76 & 30 & \multirow{4}{*}{0.7726} \\
\hline & & $\%$ & 4.36 & 25.5 & 10.07 & \\
\hline & \multirow{2}{*}{ Absent } & $\mathrm{n}$ & 19 & 121 & 39 & \\
\hline & & $\%$ & 6.38 & 40.60 & 13.09 & \\
\hline \multirow{4}{*}{ CVD } & \multirow{2}{*}{ Present } & $\mathrm{n}$ & 1 & 6 & 4 & \multirow{4}{*}{0.6027} \\
\hline & & $\%$ & 0.34 & 2.01 & 1.34 & \\
\hline & \multirow{2}{*}{ Absent } & $\mathrm{n}$ & 31 & 191 & 65 & \\
\hline & & $\%$ & 10.40 & 64.09 & 21.81 & \\
\hline \multirow{4}{*}{ Fever } & \multirow{2}{*}{ Present } & $\mathrm{n}$ & 18 & 114 & 41 & \multirow{4}{*}{0.9520} \\
\hline & & $\%$ & 6.04 & 38.26 & 13.76 & \\
\hline & \multirow{2}{*}{ Absent } & $\mathrm{n}$ & 14 & 83 & 28 & \\
\hline & & $\%$ & 4.70 & 27.85 & 9.40 & \\
\hline \multirow{4}{*}{ Cough } & \multirow{2}{*}{ Present } & $\mathrm{n}$ & 25 & 142 & 48 & \multirow{4}{*}{0.6626} \\
\hline & & $\%$ & 8.39 & 47.65 & 16.11 & \\
\hline & \multirow{2}{*}{ Absent } & $\mathrm{n}$ & 7 & 55 & 21 & \\
\hline & & $\%$ & 2.35 & 18.46 & 7.05 & \\
\hline \multirow{4}{*}{ Expectoration } & \multirow{2}{*}{ Present } & $\mathrm{n}$ & 0 & 2 & 1 & \multirow{4}{*}{0.6814} \\
\hline & & $\%$ & 0 & 0.67 & 0.34 & \\
\hline & \multirow{2}{*}{ Absent } & $\mathrm{n}$ & 32 & 195 & 68 & \\
\hline & & $\%$ & 10.74 & 65.44 & 22.82 & \\
\hline \multirow{4}{*}{ Dyspnea } & \multirow{2}{*}{ Present } & $\mathrm{n}$ & 24 & 147 & 49 & \multirow{4}{*}{0.8337} \\
\hline & & $\%$ & 8.05 & 49.33 & 16.44 & \\
\hline & & $\mathrm{n}$ & 8 & 50 & 20 & \\
\hline & & $\%$ & 2.68 & 16.78 & 6.71 & \\
\hline & & $\mathrm{n}$ & 6 & 26 & 9 & \\
\hline & & $\%$ & 2.01 & 8.72 & 3.02 & \\
\hline & & $\mathrm{n}$ & 26 & 171 & 60 & \\
\hline & & $\%$ & 8.72 & 57.38 & 20.13 & \\
\hline & & $\mathrm{n}$ & 1 & 8 & 2 & \\
\hline & & $\%$ & 0.34 & 2.68 & 0.67 & \\
\hline & & $\mathrm{n}$ & 31 & 189 & 67 & \\
\hline & & $\%$ & 10.40 & 63.42 & 22.48 & \\
\hline & & $\mathrm{n}$ & 0 & 12 & 3 & \\
\hline & & $\%$ & 0 & 4.03 & 1.01 & \\
\hline & & $\mathrm{n}$ & 32 & 185 & 66 & \\
\hline & & $\%$ & 10.74 & 62.08 & 22.15 & \\
\hline
\end{tabular}




\section{Cureus}

\begin{tabular}{|c|c|c|c|c|c|c|}
\hline \multirow{4}{*}{ Headache } & \multirow{2}{*}{ Present } & $\mathrm{n}$ & 4 & 14 & 5 & \multirow{4}{*}{0.6048} \\
\hline & & $\%$ & 1.34 & 4.7 & 1.68 & \\
\hline & \multirow{2}{*}{ Absent } & $\mathrm{n}$ & 28 & 183 & 64 & \\
\hline & & $\%$ & 9.4 & 61.4 & 21.48 & \\
\hline \multirow{4}{*}{ Anosmia } & \multirow{2}{*}{ Present } & $\mathrm{n}$ & 0 & 1 & 0 & \multirow{4}{*}{0.6605} \\
\hline & & $\%$ & 0 & 0.34 & 0 & \\
\hline & \multirow{2}{*}{ Absent } & $\mathrm{n}$ & 32 & 196 & 69 & \\
\hline & & $\%$ & 10.74 & 65.77 & 23.15 & \\
\hline \multirow{4}{*}{ Sore throat } & \multirow{2}{*}{ Present } & $n$ & 0 & 4 & 1 & \multirow{4}{*}{0.5366} \\
\hline & & $\%$ & 0 & 1.34 & 0.34 & \\
\hline & \multirow{2}{*}{ Absent } & $\mathrm{n}$ & 32 & 193 & 68 & \\
\hline & & $\%$ & 10.74 & 64.77 & 22.82 & \\
\hline \multirow{4}{*}{ Ageusia } & \multirow{2}{*}{ Present } & $n$ & 0 & 0 & 0 & \\
\hline & & $\%$ & 0 & 0 & 0 & \\
\hline & \multirow{2}{*}{ Absent } & $\mathrm{n}$ & 32 & 197 & 69 & \\
\hline & & $\%$ & 10.74 & 66.11 & 23.15 & \\
\hline \multirow{4}{*}{ Hemoptysis } & \multirow{2}{*}{ Present } & $n$ & 0 & 3 & 0 & \multirow{4}{*}{0.2867} \\
\hline & & $\%$ & 0 & 1.01 & 0 & \\
\hline & \multirow{2}{*}{ Absent } & $\mathrm{n}$ & 32 & 194 & 69 & \\
\hline & & $\%$ & 10.74 & 65.10 & 23.15 & \\
\hline
\end{tabular}

\section{TABLE 4: Correlation of CT severity categories with various variables}

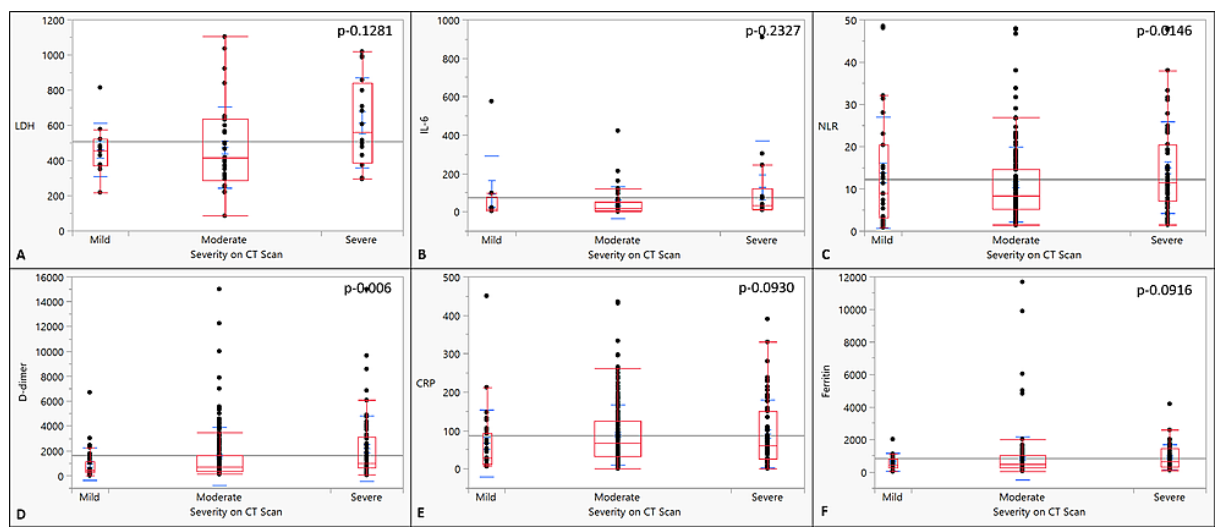

FIGURE 4: The co-relationship between severity on CT scan and inflammatory markers: (A) LDH (IU/L), (B) IL-6 (ng/dl), (C) NLR, (D) Ddimer (ng/ml), (E) CRP (mg/L), and (F) serum ferritin (ng/ml)

CRP: c-reactive protein, NLR: neutrophilic lymphocytic ratio; IL-6: interleukin-6, LDH: lactate dehydrogenase; CT score: computed tomography score

Analysis of variance (ANOVA) was used to analyze the correlation and estimate significance. 


\section{Cureus}

\begin{tabular}{|c|c|c|c|c|c|}
\hline Characteristics & & Mild & Moderate & Severe & p-value \\
\hline \multirow{3}{*}{ CRP } & $\mathrm{n}$ & 31 & 190 & 62 & \multirow{3}{*}{0.0930} \\
\hline & Median (IQR) & $29(6.2-450)$ & $66.6(0.6-434.9)$ & $60.4(0.56-390)$ & \\
\hline & Mean \pm SD & $66.52 \pm 86.98$ & $78.33 \pm 5.68$ & $88.83 \pm 11.28$ & \\
\hline \multirow{3}{*}{ LDH } & $\mathrm{n}$ & 11 & 38 & 16 & \multirow{3}{*}{0.1281} \\
\hline & Median (IQR) & $454(217-814)$ & 413(84-1103) & 560.5(293-1018) & \\
\hline & Mean \pm SD & $460.36 \pm 151.89$ & $473.66 \pm 231.09$ & $614.36 \pm 256.41$ & \\
\hline \multirow{3}{*}{ IL-6 } & $\mathrm{n}$ & 8 & 34 & 14 & \multirow{3}{*}{0.2327} \\
\hline & Median (IQR) & $15.75(3.7-575)$ & $17.62(1.32-422)$ & $31.45(9.61-909)$ & \\
\hline & Mean \pm SD & $93.33 \pm 196.95$ & $47.85 \pm 82.53$ & $128.1 \pm 242.39$ & \\
\hline \multirow{3}{*}{ Serum ferritin } & $\mathrm{n}$ & 30 & 182 & 59 & \multirow{3}{*}{0.0916} \\
\hline & Median (IQR) & $396.5(11.7-2000)$ & $464.5(37.93-11676)$ & $610(89.7-4171)$ & \\
\hline & Mean \pm SD & $588.87 \pm 550.84$ & $832.05 \pm 1329.09$ & $895.53 \pm 773.37$ & \\
\hline \multirow{3}{*}{ D-dimer } & $\mathrm{n}$ & 31 & 188 & 61 & \multirow{3}{*}{0.0006} \\
\hline & Median (IQR) & 434(3.5-6694) & $682.92(100-15000)$ & 1004(33.7-15000) & \\
\hline & Mean \pm SD & $932.56 \pm 1307.80$ & $1556.20 \pm 2330.89$ & $2182.42 \pm 2641.77$ & \\
\hline \multirow{3}{*}{ NLR } & $\mathrm{n}$ & 31 & 190 & 63 & \multirow{3}{*}{0.0146} \\
\hline & Median (IQR) & $8.92(0.83-48.48)$ & $8.40(1.36-48)$ & $11.4(1.46-47.9)$ & \\
\hline & Mean $\pm S D$ & $13.80 \pm 13.15$ & $17.00 \pm 8.8 /$ & $15.01 \pm 10.84$ & \\
\hline
\end{tabular}

\section{TABLE 5: Correlation of the CT severity categories and the inflammatory score}

CRP: c-reactive protein; LDH: lactate dehydrogenase; IL-6: interleukin-6; NLR: neutrophil-lymphocyte ratio

On survival analysis of different CTSS categories, the patients with severe CT scores had inferior survival (p0.013) (Figure 5). The 15-day overall survival percentages (15-d OS) of mild, moderate, and severe CT categories were $88.62 \%, 70.39 \%$, and $52.62 \%$, respectively, while $30 \mathrm{~d}$-OS of three categories were $59.08 \%$, $63.96 \%$, and $49.12 \%$, respectively. The median OS of the severe category was 16 days. 


\section{Cureus}

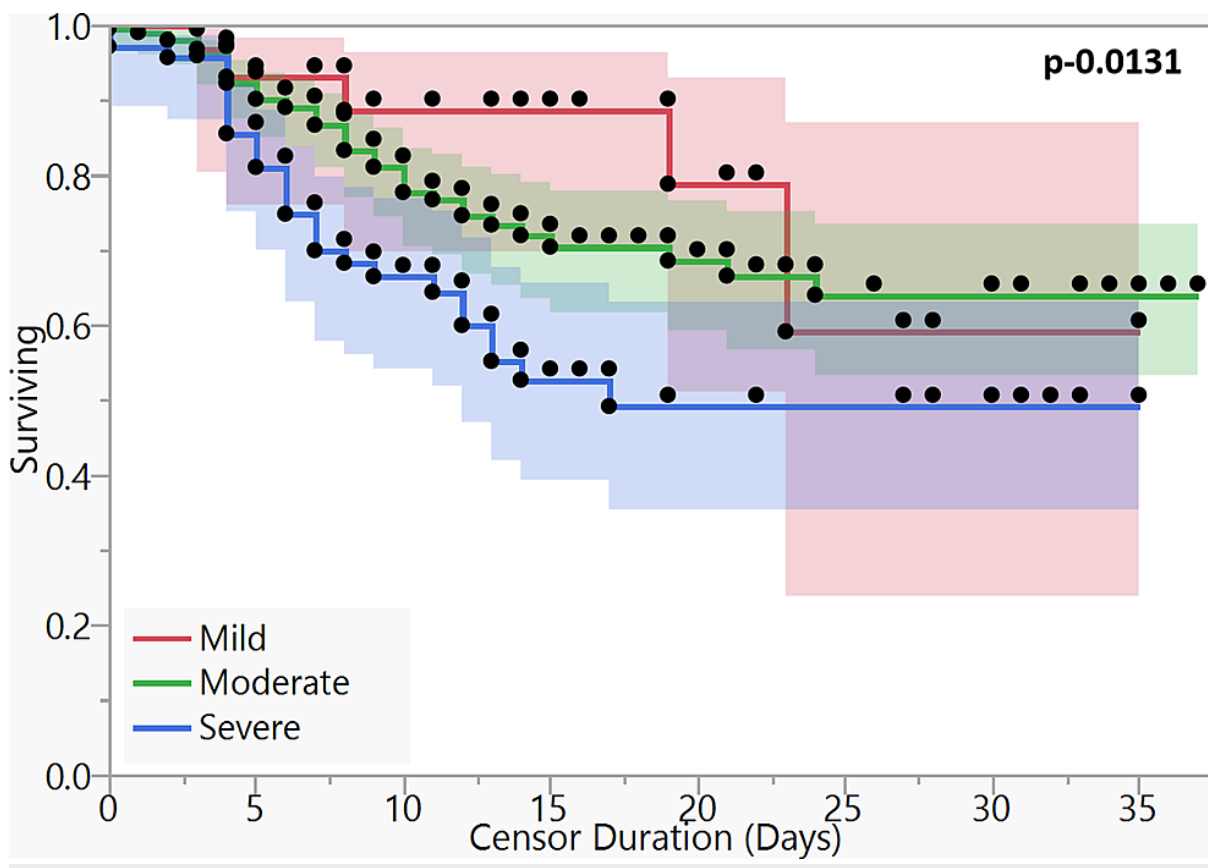

FIGURE 5: Kaplan-Meier curves for survival based on severity on CT scan

$2-8.667, \mathrm{dF}-2, \mathrm{p}-0.0131$

The log-rank test was applied to estimate significance.

\section{Discussion}

In our cohort of 298 hospitalized, hypoxemic COVID-19 patients, CT thorax showed a good correlation with 15-day and 30-day survival. The CT findings did not show any meaningful correlation with other patient demographic or laboratory parameters.

CT thorax is a good predictor of the severity of the disease [17-19]. The use of a severity score based on CT findings provides objectivity to the CT interpretation and allows correlation with other factors related to the disease process and outcomes. There is no consensus on the CT severity scoring system. A visual score used in our study has previously been shown to correlate well with disease severity. It has acceptable interobserver variability with an intragroup correlation coefficient greater than 0.9 , suggesting wider clinical applicability because of decreased subjectivity $[11,16]$. Similar findings have been obtained when automated or semi-automated software was used to analyze CT acquisition data. Compared with non-severe disease, patients with severe disease have a higher total score and higher individual scores of ground-glass opacities (GGO) consolidation. Also, the severe disease has a higher percentage of consolidation but a lower percentage of GGO relative to total lesion volume $[7,20]$. It has been shown that there is a significant difference in CT scores of scans taken in the first four days from symptom onset and those taken after Day 5 , beyond which the scores remain relatively stable in most cases, with the peak around day 10 [2,15,21]. In all our cases, a CT scan was done after a minimum of four days from symptom onset.

Our study population was different from most previous studies because all our patients had baseline hypoxia, were admitted as severe disease, and were steroid-naïve till CT thorax was conducted and blood samples were drawn. High-resolution CT thorax showed no association with any of the demographic and patient characteristics in our study. A few studies in the past have shown higher CT scores in the male population [17,22]. This finding has not been replicated in other studies [23]. Also, the denominator in these studies was remarkably different from our study as these included a large number of mild to moderate cases.

In our study, only NLR, D-dimer, and LDH had a significant correlation with the CT score. Also, only NLR and D-dimer had significant differences across CT severity score categories. A surprising finding in our study was that the CT score did not show a correlation with CRP values. This is in contrast with several previously published studies [5]. Most of these studies, however, involved patients of all severity categories. We exclusively studied patients who were admitted due to severe disease and hypoxemia. Liu et al. studied various predictors of outcome in their hospitalized COVID-19 patients. After multivariate analysis, only CT score and lymphocyte count at admission, and not CRP, were found to independently influence the outcome [24]. A study of 93 admitted patients at Wuhan showed that the CT score correlated positively with LDH, D- 
dimer, and erythrocyte sedimentation rate (ESR) but not CRP and correlated negatively with lymphocyte count, as in our study [25]. It has also been shown that in severe disease, CRP levels rise steeply in the first week of illness, even before the CT manifestations appear, and after that fall rapidly [10]. Zhang et al. showed that CRP and other laboratory parameters correlated with CT findings only in the initial stages, whereas there was no correlation of CT score with laboratory parameters thereafter [23]. CRP, thus, may be a good predictor of disease severity in the initial period, but once the disease becomes severe, the CT score may correlate better with outcomes than CRP and other inflammatory markers.

Most studies have utilized the visual scoring system used in this study, where each lobe is scored separately (from 0 to 5) and the maximum score is 25 [5,8-9,15,17,26]. Li et al. projected that a cutoff of 7 predicted severe or critical disease with a sensitivity and specificity of $80 \%$ and $82.8 \%$, respectively [26]. Francone et al. used the Kaplan-Meir analysis and projected that a CTSS equal to or more than 18 significantly increased the risk of mortality [5]. Mahdjoub reported in their study that the optimal CTSS cutoff for poor five-day outcomes was 13 [8]. Similarly, Lieveld et al. estimated that CTSS cutoffs of 10, 15, and 17 predicted hospital admission, ICU admission, and mortality, respectively, with a specificity of more than 90\% [9]. Some authors have utilized a slightly different scoring system, wherein the lungs have been divided into six zones and each zone could be scored from 0 to 4 and 24 was the maximum total score possible [12,18,21,25]. Using this system, Khosravi et al. estimated that a total score of greater than eight correlated with ICU admission, intubation, and mortality [18]. Few authors have scored each lobe from 0 to 4 and thus the total score ranged from 0 to 20 [16,24,27]. Another scoring method has been utilized by some groups, where each of 20 bronchopulmonary segments have been scored from 0 to 2 and thus the total maximum score is 40 [11]. There has been no comparison between the various scoring systems, and each of these has been shown to have an excellent inter-reader agreement [28].

Similarly, severity groups based on CTSS have varied in the literature. We divided the groups as CTSS less than 7, 7-17, and greater than 17 . This categorization has been shown to have a significant correlation with lymphopenia, CRP, ferritin, D-dimer, length of hospitalization, oxygen requirement, and ICU admission [6]. Other cutoffs for CTSS categorization have been used, like 1-5, 6-14, and 15-25 for the mild, moderate, and severe CTSS categories, respectively [29]. Rutkowska et al. arbitrarily categorized CTSS as 1-5, 6-10, 11-15, and 16-25 as mild, moderate, severe, and critical, respectively. They showed that the critical CTSS category had more lymphopenia and higher IL-6 than non-critical cases [30]. No categorization based on CTSS has been validated to be superior to others to date [28].

In this study, CT score on admission was a good predictor of outcome in terms of survival at Day 15 and Day 30. A recent review of published literature found that CT thorax at presentation has a significantly higher severity score in non-survivors compared to that in survivors, with a higher proportion of consolidations and central opacities in non-survivors [3]. Francone et al. studied the correlation of CT thorax with mortality, patient characteristics, and laboratory parameters during the initial months of the pandemic. Most patients in this study had mild disease. CT thorax showed a good correlation with mortality, CRP, and D-dimer. However, after multivariate analysis for predictors for death, only CT and age remained significant and not CRP or D-dimer [5]. Khosravi et al. recently showed that a CT score of more than eight at admission predicts a three-fold increase in the probability of ICU admission, intubation, and mortality [18].

The major strength of our study comes from the fact that we restricted our analysis specifically to severe COVID-19 patients who had hypoxia on admission. This is important because patients in this category have the worst outcomes and prognostication with CT thorax at admission may have profound implications. It is therefore noteworthy that the CT thorax maintains its prognostic prowess even among patients in the severe category. Another major strength in our study was the fact that all included patients were steroid naïve. Thus, any blunting of the inflammation due to the effect of steroids has been ruled out.

There are inherent limitations in the study due to its retrospective design. Being a single-center study, the possibility of demographic population characteristics and hospital protocols on the outcomes and study results cannot be ruled out. Interobserver variability in the interpretation of CT scores was not checked, although an excellent interobserver agreement intragroup has been described in previous studies [11,16]. All patients in our study underwent a CT scan within 24 hours of admission. However, the impact on results due to variability in duration between onset of symptoms and CT scan was not accounted for.

\section{Conclusions}

In conclusion, among hospitalized COVID-19 patients with hypoxia on admission, CT thorax severity scores correlate well with survival at 15 and 30 days. The CTSS also has a significant, although weak, correlation with NLR, D-dimer, and ferritin levels but not with CRP and IL-6. Multicenter longitudinal studies are required to confirm if CT thorax may be a better predictor of outcomes than blood inflammatory markers in hypoxic hospitalized COVID-19 patients.

\section{Additional Information}

\section{Disclosures}


Human subjects: Consent was obtained or waived by all participants in this study. Institutional Ethics Committee, Army Hospital (R\&R), Delhi Cantt issued approval 13/2021. Approved in current format. Animal subjects: All authors have confirmed that this study did not involve animal subjects or tissue. Conflicts of interest: In compliance with the ICMJE uniform disclosure form, all authors declare the following: Payment/services info: All authors have declared that no financial support was received from any organization for the submitted work. Financial relationships: All authors have declared that they have no financial relationships at present or within the previous three years with any organizations that might have an interest in the submitted work. Other relationships: All authors have declared that there are no other relationships or activities that could appear to have influenced the submitted work.

\section{References}

1. Bernheim A, Mei X, Huang M, et al.: Chest CT findings in coronavirus disease-19 (COVID-19): relationship to duration of infection. Radiology. 2020, 295:200463. 10.1148/radiol.2020200463

2. Ding X, Xu J, Zhou J, Long Q: Chest CT findings of COVID-19 pneumonia by duration of symptoms . Eur J Radiol. 2020, 127:109009. 10.1016/j.ejrad.2020.109009

3. Pontone G, Scafuri S, Mancini ME, et al.: Role of computed tomography in COVID-19. J Cardiovasc Comput Tomogr. 2021, 15:27-36. 10.1016/j.jcct.2020.08.013

4. Rubin GD, Ryerson CJ, Haramati LB, et al.: The role of chest imaging in patient management during the COVID-19 Pandemic: a multinational consensus statement from the Fleischner Society. Radiology. 2020, 296:172-80. 10.1148/radiol.2020201365

5. Francone M, Iafrate F, Masci GM, et al.: Chest CT score in COVID-19 patients: correlation with disease severity and short-term prognosis. Eur Radiol. 2020, 30:6808-17. 10.1007/s00330-020-07033-y

6. Saeed GA, Gaba W, Shah A, et al.: Correlation between chest ct severity scores and the clinical parameters of adult patients with COVID-19 pneumonia. Radiol Res Pract. 2021, 2021:6697677. 10.1155/2021/6697677

7. Sun D, Li X, Guo D, et al.: CT quantitative analysis and its relationship with clinical features for assessing the severity of patients with COVID-19. Korean J Radiol. 2020, 21:859-68. 10.3348/kjr.2020.0293

8. Mahdjoub E, Mohammad W, Lefevre T, Debray MP, Khalil A: Admission chest CT score predicts 5-day outcome in patients with COVID-19. Intensive Care Med. 2020, 46:1648-50. 10.1007/s00134-020-06118-y

9. Lieveld AW, Azijli K, Teunissen BP, et al.: Chest CT in COVID-19 at the ED: validation of the COVID-19 Reporting and Data System (CO-RADS) and CT severity score: a prospective, multicenter, observational study. Chest. 2021, 159:1126-35. 10.1016/j.chest.2020.11.026

10. Tan C, Huang Y, Shi F, et al.: C-reactive protein correlates with computed tomographic findings and predicts severe COVID-19 early. J Med Virol. 2020, 92:856-62. 10.1002/jmv.25871

11. Yang R, Li X, Liu H, et al.: Chest CT severity score: an imaging tool for assessing severe COVID-19 . Radiol Cardiothorac Imaging. 2020, 2:e200047. 10.1148/ryct.2020200047

12. Abbasi B, Akhavan R, Ghamari Khameneh A, et al.: Evaluation of the relationship between inpatient COVID-19 mortality and chest CT severity score. Am J Emerg Med. 2021, 45:458-63. 10.1016/j.ajem.2020.09.056

13. Simpson S, Kay FU, Abbara S, et al.: Radiological Society of North America expert consensus statement on reporting chest CT findings related to COVID-19. Endorsed by the Society of Thoracic Radiology, the American College of Radiology, and RSNA - secondary publication. J Thorac Imaging. 2020, 35:219-27. 10.1097/RTI.0000000000000524

14. Chang YC, Yu CJ, Chang SC, et al.: Pulmonary sequelae in convalescent patients after severe acute respiratory syndrome: evaluation with thin-section CT. Radiology. 2005, 236:1067-75. 10.1148/radiol.2363040958

15. Pan F, Ye T, Sun $P$, et al.: time course of lung changes at chest CT during recovery from coronavirus disease 2019 (COVID-19). Radiology. 2020, 295:715-21. 10.1148/radiol.2020200370

16. Li K, Fang Y, Li W, et al.: CT image visual quantitative evaluation and clinical classification of coronavirus disease (COVID-19). Eur Radiol. 2020, 30:4407-16. 10.1007/s00330-020-06817-6

17. Al-Mosawe AM, Abdulwahid HM, Fayadh NA: Spectrum of CT appearance and CT severity index of COVID19 pulmonary infection in correlation with age, sex, and PCR test: an Iraqi experience. Egypt J Radiol Nucl Med. 2021, 52:40. 10.1186/s43055-021-00422-3

18. Khosravi B, Aghaghazvini L, Sorouri M, et al.: Predictive value of initial CT scan for various adverse outcomes in patients with COVID-19 pneumonia. Heart Lung. 2021, 50:13-20. 10.1016/j.hrtlng.2020.10.005

19. Zheng Y, Wang L, Ben S: Meta-analysis of chest CT features of patients with COVID-19 pneumonia . J Med Virol. 2021, 93:241-9. 10.1002/jmv.26218

20. Salvatore C, Roberta F, Angela L, et al.: Clinical and laboratory data, radiological structured report findings and quantitative evaluation of lung involvement on baseline chest CT in COVID-19 patients to predict prognosis. Radiol Med. 2021, 126:29-39. 10.1007/s11547-020-01293-W

21. Wang Y, Dong C, Hu Y, et al.: Temporal changes of CT findings in 90 patients with COVID-19 pneumonia: a longitudinal study. Radiology. 2020, 296:E55-64. 10.1148/radiol.2020200843

22. Shang Y, Xu C, Jiang F, et al.: Clinical characteristics and changes of chest CT features in 307 patients with common COVID-19 pneumonia infected SARS-CoV-2: a multicenter study in Jiangsu, China. Int J Infect Dis. 2020, 96:157-62. 10.1016/j.ijid.2020.05.006

23. Zhang B, Zhang J, Chen H, et al.: Novel coronavirus disease 2019 (COVID-19): relationship between chest CT scores and laboratory parameters. Eur J Nucl Med Mol Imaging. 2020, 47:2083-9. 10.1007/s00259-02004854-3

24. Liu Z, Jin C, Wu CC, et al.: Association between initial chest CT or clinical features and clinical course in patients with coronavirus disease 2019 pneumonia. Korean J Radiol. 2020, 21:736-45. 10.3348/kjr.2020.0171

25. Li L, Yang L, Gui S, et al.: Association of clinical and radiographic findings with the outcomes of 93 patients with COVID-19 in Wuhan, China. Theranostics. 2020, 10:6113-21. 10.7150/thno.46569

26. Li K, Wu J, Wu F, Guo D, Chen L, Fang Z, Li C: The clinical and chest CT features associated with severe and 


\section{Cureus}

critical COVID-19 pneumonia. Invest Radiol. 2020, 55:327-31. 10.1097/RLI.0000000000000672

27. Lyu P, Liu X, Zhang R, Shi L, Gao J: The performance of chest CT in evaluating the clinical severity of COVID-19 pneumonia: identifying critical cases based on CT characteristics. Invest Radiol. 2020, 55:412-21. 10.1097/RLI.0000000000000689

28. Wasilewski PG, Mruk B, Mazur S, Półtorak-Szymczak G, Sklinda K, Walecki J: COVID-19 severity scoring systems in radiological imaging - a review. Pol J Radiol. 2020, 85:e361-8. 10.5114/pjr.2020.98009

29. Tabatabaei SM, Rajebi H, Moghaddas F, Ghasemiadl M, Talari H: Chest CT in COVID-19 pneumonia: what are the findings in mid-term follow-up?. Emerg Radiol. 2020, 27:711-9. 10.1007/s10140-020-01869-z

30. Rutkowska E, Kwiecień I, Żabicka M, et al.: Cytokines and leukocytes subpopulations profile in SARS-CoV-2 patients depending on the CT score severity. Viruses. 2021, 13:880. 10.3390/v13050880 